\title{
Influence of Technical Improvements on the Business Case for a Mobile WiMAX Network
}

\author{
Bart Lannoo, Jeffrey De Bruyne, Wout Joseph, Jan Van Ooteghem, \\ Emmeric Tanghe, Didier Colle, Luc Martens, Mario Pickavet, and Piet Demeester \\ Dept. of Information Technology (INTEC), Ghent University - IBBT, \\ Gaston Crommenlaan 8 box 201, 9050 Gent, Belgium \\ \{bart.lannoo, jeffrey.debruyne, wout.joseph, jan.vanooteghem, \\ emmeric.tanghe, didier.colle, luc.martens, mario.pickavet, \\ piet.demeester\}@intec.ugent.be
}

\begin{abstract}
From a technical point of view, Mobile WiMAX may offer an appropriate solution for delivering broadband wireless access. Two remaining questions, however, are whether the rollout of a WiMAX network is economically feasible or not, and how technical improvements like MIMO, beamforming and turbo-coding can influence the business case. In this paper different technical scenarios for a Mobile WiMAX rollout are defined and evaluated from an economic as well as a technical perspective. To obtain realistic figures, we have defined a business case for a Mobile WiMAX rollout in Belgium, a country with a high wired broadband penetration. Further, we give an indication about the feasibility of a Mobile WiMAX network related to the population density. Finally, as the introduction of a new technology involves a lot of uncertainties, a detailed sensitivity analysis on both the economic and technical input parameters is performed to determine the most influencing parameters.
\end{abstract}

Keywords: Broadband Wireless Communication, WiMAX, 802.16e-2005, Mobility, Business Case, Techno-economic Analysis

\section{Introduction}

Ever-increasing bandwidth demands and recent mobility trends are two main challenges for the access communications network during the next years. Based on the booming markets of broadband connectivity and mobile phone usage, it may be clear that there exists a great potential for broadband services on mobile terminals. To enhance mobility in the access network, Mobile WiMAX may possibly offer an appropriate solution. Its bandwidth is high enough to be a competing technology for the currently available fixed access networks (like e.g., DSL, cable), and by its support for mobility, it can also be an alternative for mobile access networks (like e.g., UMTS, HSDPA). Note that WiMAX is a certification label, mainly based on the IEEE 802.16 standards [1-3] and promoted by the WiMAX Forum [4]. Today, two important WiMAX profiles are defined: a Fixed and Mobile version. Due to the 
addition of mobility, it is expected that mainly Mobile WiMAX (based on the IEEE 802.16e-2005 standard [3]) will be used in the future.

The performance of Mobile WiMAX systems has already been extensively investigated [5-7]. Coverage and performance of Mobile WiMAX have been analyzed via link and system simulations in [5-6], and [7] presents an overview of some multiple antenna technologies like Multiple Input Multiple Output (MIMO) and beamforming, which have gained a lot of interest the latest years because of their potential benefits in increasing coverage or capacity. Next to these technical studies, the economic feasibility of a Mobile WiMAX deployment has also already been investigated. In [8-9], results are given for a developed country (Belgium) with a high penetration of a wired infrastructure (DSL and cable). These two papers especially focus on the economic feasibility of Mobile WiMAX for outdoor reception and the sensitivity of the business case is only applied to uncertainties in the economic parameters (i.e. costs, revenues and user adoption). Technical parameters such as antenna heights, path loss, MIMO and beamforming gains, and penetration loss also include a high uncertainty, and, by our knowledge, no research up to now has been performed about the influence of this uncertainty on the economic viability of a Mobile WiMAX rollout. In this paper, we present the results of an extensive analysis about the rollout of Mobile WiMAX for both outdoor and indoor coverage in Belgium. For this analysis, we have developed a planning tool which incorporates all necessary economic and technical aspects. A thorough sensitivity analysis is also executed to compare the influence of technical and economic parameters.

The most important characteristics of a Mobile WiMAX system and the related business case are described in Section 2. Section 3 shows the results of the link budget calculations for Mobile WiMAX, which is necessary to obtain the number of required base station (BS) sites to cover a certain area. Different technical solutions and propagation models are compared to each other. Section 4 discusses the influence of different technical characteristics on the rollout of Mobile WiMAX (for outdoor and indoor coverage) in Belgium by means of a net present value (NPV) analysis. Section 5 presents a detailed sensitivity analysis to indicate the most influencing parameters within our model. Finally, conclusions are presented in Section 6.

\section{Configuration}

Mobile WiMAX typically uses a cellular approach, requiring the installation of several WiMAX cell sites or BSs through the covered area. The number of needed BSs is a determining cost factor in a WiMAX deployment. That is why it is very important to properly dimension the network. An accurate planning tool has been developed which incorporates all the necessary technical and economic aspects that will be discussed in the following sections.

\subsection{Selection of Characteristics of Mobile WiMAX System}

We describe the physical characteristics of a Mobile WiMAX system and motivate the selected parameter values of our reference Mobile WiMAX system. As some 
technologies will improve in the future, some additional gains are also considered. These characteristics are vendor-specific, which indicates the importance of a sensitivity analysis including these technical parameters as well (discussed in Section 5).

WiMAX System Characteristics. Deployments of $802.16 \mathrm{e}$ are preferred in the frequency band between $2.3 \mathrm{GHz}$ and $2.5 \mathrm{GHz}$ [10]. In this paper we consider a Time Division Duplex (TDD) system (3:1 downlink (DL) / uplink (UL) ratio) with a carrier frequency of $2.5 \mathrm{GHz}$, channel bandwidth of $10 \mathrm{MHz}$ and cyclic prefix of $1 / 8$. The system also provides adaptive modulation from QPSK 1/2 to 64-QAM 3/4, which corresponds with bitrates ranging from 4 to $19 \mathrm{Mbps}$ for DL, and 1 to $5 \mathrm{Mbps}$ for UL (considering a protocol overhead of $20 \%$ ).

A BS antenna height of $30 \mathrm{~m}$, a BS transmit power of $35 \mathrm{dBm}$ and a BS antenna gain of $16 \mathrm{~dB}$ are chosen for our analysis. For the subscriber station (SS), we consider a height of $2 \mathrm{~m}$, a transmit power of $27 \mathrm{dBm}$, and an antenna gain of $2 \mathrm{~dB}$, which is equal to the gain of a realistic dipole antenna (typically used for SSs such as laptops).

Receiver Sensitivity. The required receiver $(\mathrm{Rx})$ sensitivity to detect the wireless signal at the SS (DL) or BS (UL) is an essential parameter for determining the range of the Mobile WiMAX system. This minimum Rx sensitivity is vendor-specific and can be calculated from the thermal noise $(-174 \mathrm{dBm}$, at $290 \mathrm{~K})$, the receiver Signal-toNoise ratio (SNR), the receiver noise figure $(5 \mathrm{~dB}$ in $\mathrm{UL}, 7 \mathrm{~dB}$ in $\mathrm{DL})$ and the implementation loss $(2 \mathrm{~dB})[10]$. The SNR depends on the modulation scheme and Forward Error Correction (FEC) algorithm. Two much used FEC algorithms are the Reed-Solomon Convolutional Code (RS-CC) and the Convolutional Turbo Code (CTC). RS-CC has higher SNR requirements than CTC, but CTC from its side requires a higher implementation cost.

Multiple Antenna Techniques. To improve radio performance, multiple antenna technologies are very often used in cellular systems. Currently, more advanced antenna techniques such as smart antenna technology with MIMO and beamforming antennas are proposed.

MIMO: MIMO systems refer to solutions where the same information is transmitted (after space-time coding) in space and time. The theoretical diversity gain of such a solution for both DL and UL is a function of the product of the transmit/receive antennas and is equal to $10 \log \left(\mathrm{N}_{\mathrm{Tx}} \times \mathrm{N}_{\mathrm{Rx}}\right)$ [11], with $\mathrm{N}_{T \mathrm{x}}$ the number of transmit antennas and $\mathrm{N}_{\mathrm{Rx}}$ the number of receive antennas. Furthermore, the use of MIMO also includes a Cyclic Combining Gain of $3 \mathrm{~dB}$ in DL [5].

Beamforming or Adaptive Antenna System (AAS): The beamforming technology principle is to coherently combine the signals received from $\mathrm{N}$ antenna elements of an antenna array. Beamforming improves the link budget for the data transmission for both the DL and UL. Indeed, by concentrating the energy in one direction, the resulting antenna gain in one direction is significantly increased. This additional gain is beneficial for improving the coverage of the $\mathrm{BS}$ and/or reducing the power needed by the SS to transmit signals. Theoretical gains (in comparison to a conventional 
antenna) for an N-element antenna array are $20 \log (\mathrm{N})$ for DL and $10 \log (\mathrm{N})$ for UL [11].

Orthogonal Frequency Division Multiple Access (OFDMA). In an OFDMA system, resources are available in the time domain by means of OFDM symbols and in the frequency domain by means of sub-carriers. The time and frequency resources can then be organized into subchannels for allocation to individual users (as well in DL as in UL). This usage of subchannels causes a subchanneling gain which is equal to $-10 \log$ (Fraction of Number of Used Subchannels to total number of Available Subchannels) [12]. In this paper we consider only uplink subchanneling gain.

\subsection{Selection of Characteristics of Business Case}

Different business parameters (rollout, services, tariffs) are selected by the operator and others (equipment costs) are vendor-specific. Combined with a chosen rollout area and well-estimated market forecast, an economic feasibility study can be performed for a Mobile WiMAX rollout (see Section 4).

Possible Rollout Scenarios. The optimal rollout strategy of a new WiMAX network typically depends on the targeted market. In developing countries with a low penetration of fixed access networks, WiMAX can be a viable alternative for DSL or cable access. In developed countries, WiMAX can be a complementary technology with the current access networks, providing the advantage of portability or even mobility. In this paper, we especially focus on a Mobile WiMAX deployment in a developed country, starting in the urban areas since they have the largest potential customer base.

A second important part in the rollout strategy of an operator is the assumed rollout speed. Due to practical constraints and/or too high investments, it may be preferred to spread a full (e.g., nationwide) rollout over several years. Otherwise, for a booming market with a lot of strong competitors, it may be advantageous to adopt a faster rollout speed to gain a first-mover advantage. The preferred strategy however, is very case-specific, and in Section 4, we discuss a case in Belgium.

Offered Services. To reach as much customers as possible, an operator has to differentiate its offered products, each corresponding to a varying user profile or target group. In this way, we assume four service types for broadband Internet access (summarized in Table 2): 'Stand-alone wireless broadband' (WiMAX used as broadband connection, instead of DSL or cable, especially suited for the residents of the covered area), 'Second residence' (mainly intended for users that need a second connection, e.g., business people, students), 'Nomadicity' (a light version of the previous product, comparable with the current subscriptions to a hotspot, suited for a varying target group) and 'Prepaid' (a prepaid card grants the user a limited number of hours for using the WiMAX network, used by e.g., tourists).

We suppose that the 'Stand-alone' and 'Second residence' service offer a bandwidth of $3 \mathrm{Mbps}$ downstream and $256 \mathrm{kbps}$ upstream, and the other two services 
have a bandwidth of $512 \mathrm{kbps}$ downstream and $128 \mathrm{kbps}$ upstream. Furthermore, we assume an overbooking factor of 20 as it will never occur that every subscriber simultaneously uses its full WiMAX connection.

Tariff Setting. A good tariff setting is extremely important as it defines the final profits of an operator. If the tariffs are set too low, the operator will lose a lot of revenues, but on the other hand, a too high tariff will have a negative effect on the adoption. The assumed tariffs in our analysis are shown in Table 2, and are comparable to similar products, currently offered by diverse operators.

CapEx and OpEx. Capital Expenditures (CapEx) contain the rollout costs of the new WiMAX network. An important equipment cost originates from the WiMAX BSs, of which the antenna cost depends on the chosen multiple antenna technique. The BSs are installed on pylons or on rooftops of existing buildings. Reusing existing pylons is very often stimulated or even regulated, and then only a rental price is required, just as for placing an antenna on a rooftop. Building a new pylon on the other hand requires a high investment cost. In addition, costs for connecting the BSs to the backhaul network (e.g., fiber network or backhauling via WiMAX) and costs for core equipment in the central office are required. Equipment is renewed every five years (economic and technical lifetime) and a yearly cost erosion of $5 \%$ is taken into account.

Operational Expenditures (OpEx) contain the yearly returning costs and they can be divided between network and service related costs. The former consists of costs for operations \& planning, maintenance, leasing of the sites (if applicable, e.g., pylons) and backhauling. The latter incorporate costs for marketing, sales \& billing and helpdesk.

Regarding the WiMAX spectrum license costs, they can be considered as either CapEx or OpEx, but this is very country-specific and determined by the national telecom regulator.

\subsection{Overview}

Table 1 and Table 2 summarize the technical characteristics of a Mobile WiMAX network and the offered service types, respectively.

We define three main technical scenarios:

- $\quad$ Reference scenario (Table 1): Typical commercial systems currently available.

- Extended scenario 1: 'Reference scenario' extended with two more antennas for MIMO at BS side (so $4 \times 2$ in DL, and $1 \times 4$ in UL).

- Extended scenario 2: 'Reference scenario' extended with two more antennas for MIMO at BS side (so $4 \times 2$ in DL, and $1 \times 4$ in UL) and 4 -antenna array AAS at $\mathrm{BS}$ side.

Each of the three above scenarios is separated in two subscenarios: one using RSCC as FEC algorithm, the other using CTC (both are already available in commercial systems). 
Table 1. Parameter values of Mobile WiMAX characteristics of reference scenario [5], [10-13].

\begin{tabular}{|c|c|}
\hline Parameter & Value \\
\hline \multicolumn{2}{|l|}{ Mobile WiMAX System } \\
\hline Carrier Frequency & $2.5 \mathrm{GHz}$ \\
\hline Channel Bandwidth & $10 \mathrm{MHz}$ \\
\hline Duplexing & TDD \\
\hline $\mathrm{DL} / \mathrm{UL}$ ratio & $3: 1$ \\
\hline FEC & $\mathrm{RS}-\mathrm{CC}$ \\
\hline Cyclic Prefix (CP) & $1 / 8$ \\
\hline \multicolumn{2}{|l|}{ BS Characteristics } \\
\hline BS Height & $30 \mathrm{~m}$ \\
\hline Tx (DL) output power & $35 \mathrm{dBm}(3.2 \mathrm{~W})$ \\
\hline $\mathrm{Tx}(\mathrm{DL}) / \mathrm{Rx}(\mathrm{UL})$ antenna gain & $16 \mathrm{~dB}$ \\
\hline Tx (DL) / Rx (UL) feeder loss & $0.5 \mathrm{~dB}$ \\
\hline Noise Figure (UL) & $5 \mathrm{~dB}$ \\
\hline Implementation Loss (UL) & $2 \mathrm{~dB}$ \\
\hline \multicolumn{2}{|l|}{ Mobile SS Characteristics } \\
\hline SS Height & $2 \mathrm{~m}$ \\
\hline Tx (UL) output power & $27 \mathrm{dBm}(0.5 \mathrm{~W})$ \\
\hline $\mathrm{Tx}(\mathrm{UL}) / \mathrm{Rx}(\mathrm{DL})$ antenna gain & $2 \mathrm{~dB}$ \\
\hline Tx (UL) / Rx (DL) feeder loss & $0 \mathrm{~dB}$ \\
\hline Noise Figure (DL) & $7 \mathrm{~dB}$ \\
\hline Implementation Loss (DL) & $2 \mathrm{~dB}$ \\
\hline \multicolumn{2}{|l|}{ Uplink Subchanneling } \\
\hline \multirow{2}{*}{ Gain } & _10log used subchannels \\
\hline & max (\# subchannels) \\
\hline \multicolumn{2}{|l|}{$\begin{array}{l}\text { MIMO: Reference scenario } \\
(2 \times 2 \text { in DL, 1x2 in UL) }\end{array}$} \\
\hline MIMO gain DL /UL & $6 \mathrm{~dB} / 3 \mathrm{~dB}$ \\
\hline Cyclic combining gain (DL) & $3 \mathrm{~dB}$ \\
\hline
\end{tabular}

Table 2. Parameter values of offered Mobile WiMAX services.

\begin{tabular}{lccc}
\hline \multicolumn{1}{c}{ Parameter } & \multicolumn{2}{c}{ Value } \\
\hline \multirow{2}{*}{ Offered service } & Tariff (incl. VAT) & \multicolumn{2}{c}{ Offered bit rate } \\
\cline { 3 - 4 } & $13 € /$ month & Downlink & Uplink \\
\hline Nomadicity & $23 € /$ month & $3 \mathrm{kbps}$ & $128 \mathrm{kbps}$ \\
Second residence & $9 € / 3$-hour card & $512 \mathrm{kbps}$ & $256 \mathrm{kbps}$ \\
Prepaid & $40 € /$ month & $3 \mathrm{Mbps}$ & $256 \mathrm{kbps}$ \\
Stand-alone wireless broadband & & & \\
\hline
\end{tabular}




\section{Link Budget and Coverage Range}

The calculation and tabulation of signal powers, gains, losses and SNR for a complete communication link is called a link budget, which is a useful approach for the basic design of a communication system. Using the link budget parameters, the coverage range of the Mobile WiMAX system can be obtained for different modulation schemes. These coverage ranges are necessary to obtain the required number of BSs for the rollout of a Mobile WiMAX network.

Next to the vendor-specific parameters described in Section 2.1, other technical parameters such as path loss, penetration loss (for indoor coverage) and several margins are also necessary for the link budget calculations. These parameters are described in the following paragraphs. Using the technical scenarios introduced in Section 2.3, the results of the coverage ranges for different modulation schemes are shown.

\subsection{Path Loss Models}

The path loss is defined as the transmit power times the antenna gains divided by the mean received power. Different path loss or propagation models have been defined, and a comparison of them can be found in [5], [13].

The first one is the COST-231 Hata model which is based on empirical results in the $2 \mathrm{GHz}$ band and tends to make very conservative coverage predictions for $2.5 \mathrm{GHz}$. A second model is the COST-231 Walfisch-Ikegami (W-I) model matching extensive experimental data for flat suburban and urban areas with uniform building height. The COST-231 W-I model gives more precise path loss than the Hata model due to additional data parameters: building heights, street width, building separation and street orientation with respect to the direct radio path. The third one is the Erceg model, which is based on extensive experimental data collected at $1.9 \mathrm{GHz}$ for suburban areas in the US [14], and which has been expanded with a correction factor to cover higher frequencies. The Erceg model has three variants (A, B, and C) based on the terrain type, varying from very hilly terrains with heavy tree density (type A) to flat terrains with low tree density (type C). This model is recommended for Fixed and Mobile WiMAX link budget calculations [5].

\subsection{Building Penetration Loss}

The received power at indoor locations is significantly attenuated depending on the materials and the construction of the buildings. Several measurements have been carried out to verify real values of attenuation. A large spread of building penetration losses have been obtained in [15] and the practical range varies from $7 \mathrm{~dB}$ to $15 \mathrm{~dB}$. For our research, we assume an average building penetration loss of $11 \mathrm{~dB}$ with a standard deviation of $6 \mathrm{~dB}$. 


\subsection{Shadowing, Fade and Interference Margin}

Shadowing Margin. A service (offered by the network operator) should have to be provided at more than $90 \%$ of all locations within a cell with $99 \%$ reliability. To achieve this, a shadowing margin is necessary for accounting the temporary variations in signal strength caused by trees, buildings, etc. located on the signal path between transmitter and receiver. This shadowing margin depends on the used path loss model. For the Erceg model [14], shadowing margins of $13.6 \mathrm{~dB}$ (A), $12.3 \mathrm{~dB}$ (B), and $10.5 \mathrm{~dB}(\mathrm{C})$ are obtained for a coverage requirement of $90 \%$ at the edge of the cell. For the Hata and W-I model we use shadowing margins of $10 \mathrm{~dB}$ [13].

Note that the shadowing margin at indoor locations is the combined result of the outdoor variation and the variation due to building attenuation. As a consequence, the shadowing margin increases for indoor reception.

Fade Margin. The fade margin takes the yearly availability of the system into account. The link availability is affected by clear-air and rain multipath fading. We use the ITU-R P.530 model described in the ECC report [16] for the fade margin. A fade margin of $10 \mathrm{~dB}$ is used which results in a yearly availability of $99.995 \%$ for a cell radius of $10 \mathrm{~km}$.

Interference Margin. The system's performance is limited by co-channel interference (CCI). The most common sources of $\mathrm{CCI}$ are due to frequency reuse: inter-cell interference (at the cell edges) and intra-cell interference (at the sector boundaries). [5] proposes maximum interference margins of $2 \mathrm{~dB}$ and $3 \mathrm{~dB}$ for $\mathrm{DL}$ and UL, respectively and are also used in our analysis.

\subsection{Coverage Range Results}

Fig. 1 shows the ranges in DL for different path loss models, outdoor and indoor environments, and BS heights in urban areas with gains and margins described in previous sections (see also Table 1). Note that we include an additional $3 \mathrm{~dB}$ margin for Erceg $\mathrm{C}$ in urban areas, as this is also used for the COST-231 Hata model in urban areas.

Fig. 1 shows that for the reference scenario (Table 1), the path loss model of Erceg type $\mathrm{C}$ delivers ranges of $1300 \mathrm{~m}$ and $600 \mathrm{~m}$ for QPSK $1 / 2$ and 64-QAM 3/4, respectively. COST-231 Hata and W-I give significantly lower ranges than Erceg type C (e.g., only $500 \mathrm{~m}$ can be reached for QPSK 1/2). Upgrading the technology to 'Extended scenario 2 with CTC' improves considerably the ranges (e.g., from $1300 \mathrm{~m}$ to $3300 \mathrm{~m}$ for QPSK 1/2, using Erceg type C). This motivates the use of technologies such as MIMO and beamforming.

Indoor reception is also of great importance for the usage of Mobile WiMAX applications. Fig. 1 shows the results for indoor reception for 'Extended scenario 2 with CTC', and we observe a significant decrease in range (e.g., from $3300 \mathrm{~m}$ to $1500 \mathrm{~m}$ for QPSK 1/2). This will have a high influence on the business case of a 
Mobile WiMAX rollout, especially when the operator wants to guarantee mobile connection at any time at any place. A solution to enhance the coverage range is the use of more additional antennas and/or changing the BS antenna height. If we move the antenna from $30 \mathrm{~m}$ to $45 \mathrm{~m}$, we can observe in Fig. 1 an increase in range from $1500 \mathrm{~m}$ to $1900 \mathrm{~m}$ for QPSK $1 / 2$.

These results indicate the strong influence of the technical parameters on the range and by consequence on the minimum required base stations for a Mobile WiMAX deployment.

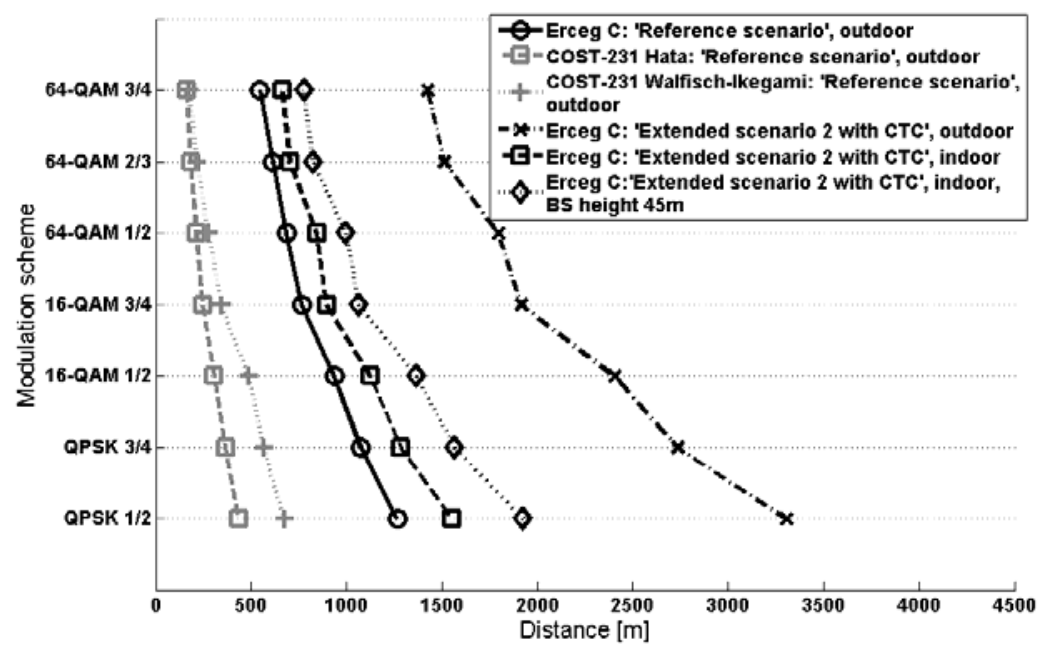

Fig. 1. Coverage range results in downlink for different technical scenarios in urban areas (outdoor and indoor).

\section{Economic Feasibility of a Mobile WiMAX Rollout}

In this section, we analyze the feasibility of a realistic business case for Mobile WiMAX in the period 2008-2017 in Belgium (counting 10.6 million inhabitants on an area of $30,528 \mathrm{~km}^{2}$ ). The different technical scenarios (described in Section 2.3) are compared to each other, using Erceg $\mathrm{C}$ as path loss model, because of Belgian flat nature.

For our analysis, we assume an S-shaped adoption curve following the Gompertz model, with different input parameters for the four services. Because of the already high penetration of wired access networks in Belgium, Mobile WiMAX will probably not succeed as 'Stand-alone' service, but more as a complementary service ('Nomadicity' and 'Second residence'). We assume for the subscriptions to the 'Stand-alone', 'Nomadicity' and 'Second residence' services, final adoptions of ca. $0.5 \%, 8.5 \%$, and $5.0 \%$ of the households, respectively. Besides, the number of yearly sold prepaid cards corresponds to ca. $3.5 \%$ of the Belgian inhabitants (inh). Note 
however that especially the 'Second residence' service is not only intended for covered households, and that prepaid cards can also be bought by foreigners. So, the above numbers give only a rough indication about the adoption, more precise details are given in [9].

\subsection{Determining the Optimal Rollout Area}

For a first analysis, we suppose that the network rolled out is deployed in one year, and compare the impact of an increasing number of municipalities where the network is deployed. The rollout sequence is based on population density. In this way, it is possible to estimate the minimal required density to obtain a positive business case for both outdoor and indoor coverage.

Fig. 2 shows the NPV results after ten years (taking into account a discount rate of $15 \%$ ) for the six technical scenarios, assuming outdoor coverage. The $\mathrm{X}_{1}$-axis indicates the number of municipalities that is covered, and the $\mathrm{X}_{2}$-axis represents the corresponding population density. We see that the reference scenario for outdoor coverage generates a positive NPV from a population density of $1000 \mathrm{inh} / \mathrm{km}^{2}$. We also notice that it is most advantageous to extend the coverage area to all cities with a minimum of $1800 \mathrm{inh} / \mathrm{km}^{2}$ (where a maximum NPV of $25 \mathrm{M} €$ is obtained). Further, an evolving technology has a very positive influence on the business case leading to a shift of the curves to the right, and the maximum NPV increases up to $50 \mathrm{M} €$ for the most optimistic scenario ('Extended scenario 2 with CTC' for a deployment in an area with a minimum population density of $1000 \mathrm{inh} / \mathrm{km}^{2}$ ). For this scenario, a rollout in almost all suburban areas (i.e. up to $400 \mathrm{inh} / \mathrm{km}^{2}$ ) is feasible.

\section{Outdoor coverage}

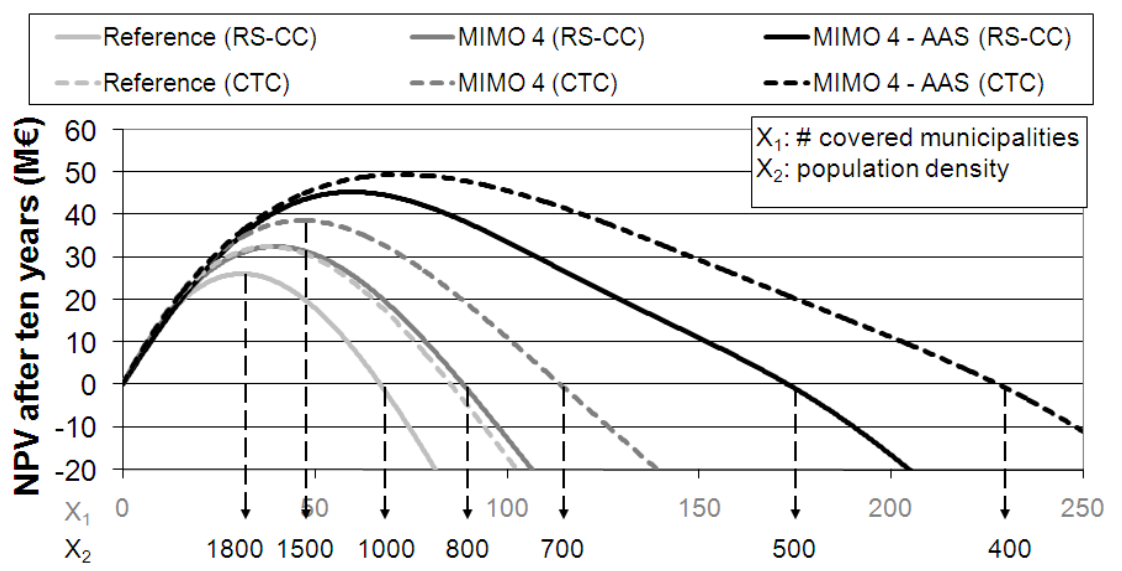

Fig. 2. NPV results for outdoor coverage for six different technical scenarios.

Fig. 3 shows that indoor coverage has a very high influence on the business case. With the assumed adoption, indoor coverage is only feasible for a rollout in very 
densely populated areas: above $5000 \mathrm{inh} / \mathrm{km}^{2}$ for the reference scenario, and for minimum $1700 \mathrm{inh} / \mathrm{km}^{2}$ for the most optimistic technical scenario. Most likely however, adoption will change if indoor coverage is guaranteed, but it turns out that the adoption has to be increased by a factor of 4 to 5 to obtain a business case comparable to outdoor coverage.

Indoor coverage

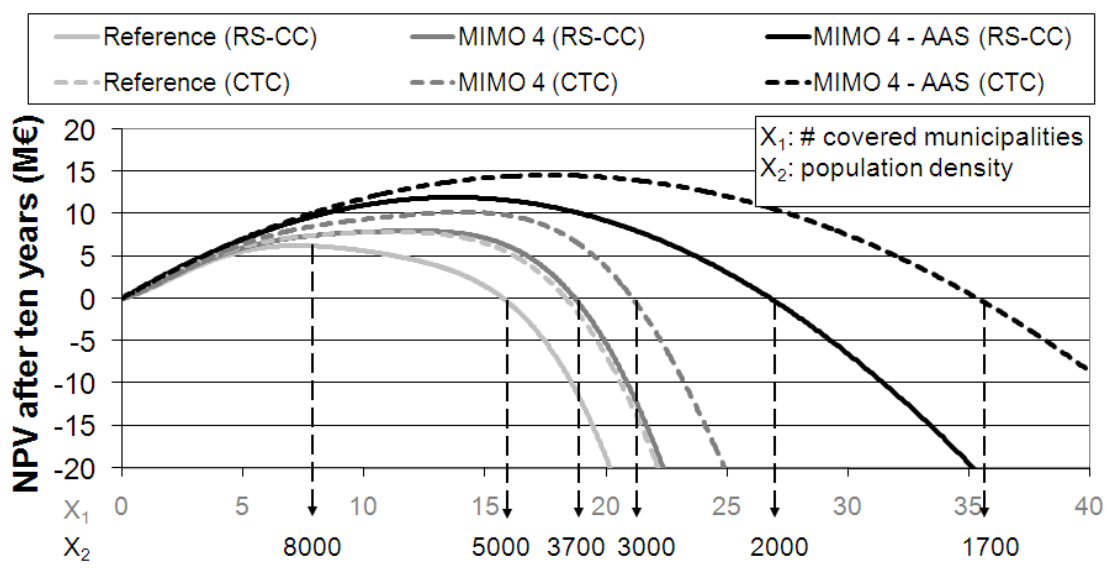

Fig. 3. NPV results for indoor coverage for six different technical scenarios.

\subsection{Detailed Analysis for a 5-Year Rollout}

As mentioned in Section 2.2, a realistic rollout is typically spread over several years. More details about the most suited rollout speed are provided in [9]. Fig. 4 shows the outcome of a 5-year rollout in Belgium providing outdoor coverage in all areas with $1000 \mathrm{inh} / \mathrm{km}^{2}$ or more (corresponding to $8 \%$ of the area and $36 \%$ of the population of Belgium). The three technical scenarios with RS-CC as FEC algorithm are compared to each other and the NPV in 2017 matches very well to the corresponding points on Fig. 2. Next to the NPV results, the number of required BSs after each year is also depicted, as this is the main factor responsible for the differences between the technical scenarios. Upgrading the technology from our reference scenario to the 'Extended scenario 2' leads to a 30\% decrease of the number of required BSs. 


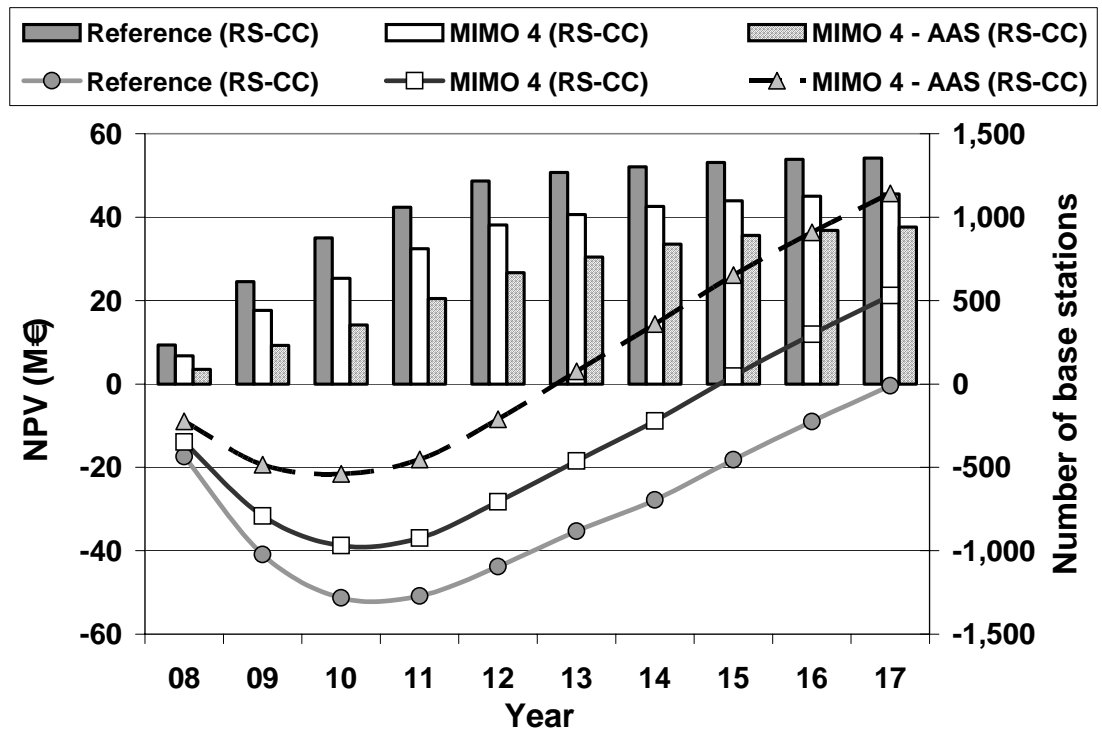

Fig. 4. NPV results (lines) and number of required base stations (columns) for a realistic 5-year rollout and outdoor coverage.

\section{Sensitivity Analysis}

A detailed sensitivity analysis by using Monte Carlo simulations is performed to assess the potential impact of uncertainties on the project's outcome. The aim is to identify the impact of changes in both technical and economic assumptions.

\subsection{Varying Parameters}

This section discusses the considered input parameters.

Technical Parameters. A general parameter for link budget variations and the base station heights are two technical input parameters that are varied in our sensitivity analysis.

Link Budget Parameters. A lot of physical characteristics such as the admitted transmit power, antenna gains, additional gains caused by MIMO and AAS, and required SNR are vendor-specific, and can be improved in the future. We have varied the total link budget for our analysis according to a Gaussian distribution with a standard deviation of $1 \mathrm{~dB}$. 
Base Station Height. In Section 4 we consider a fixed BS height of $30 \mathrm{~m}$, but this height will of course vary for different municipalities. For our sensitivity analysis, the base station height is varied according to a Gaussian distribution with a mean value of $30 \mathrm{~m}$, and a standard deviation of $10 \%$.

Economic parameters. Different economic input parameters like adoption, costs and revenues, together with the offered services are also considered in our sensitivity analysis.

Adoption, CapEx and OpEx, and Service Tariffs. These are the most important economic parameters and for our sensitivity analysis, we have varied them according to a Gaussian distribution, with a standard deviation of $10 \%$. Note that especially the adoption can be very uncertain, but for a fair comparison we have equally varied the four parameters. In [9], the sensitivity analysis is limited to these four economic parameters.

Offered Bit Rates. Like the service tariff (see Section 2.2), the bit rate for each service is set by the operator. To compare its influence with the other parameters, we have varied it according to a Gaussian distribution, with a standard deviation of $1 \mathrm{Mbps}$ for a $3 \mathrm{Mbps}$ service (and proportionally for the other bit rates).

\subsection{Results}

Fig. 5 shows the sensitivity results of ten consecutive years for the 5-year rollout scenario. For each year the sensitivity results of different input parameters are analyzed.

One very important conclusion from Fig. 5 is that, during the five rollout years (2008 to 2012), the NPV is more sensitive to both technical parameters than to the economic ones. The link budget and BS heights together experience a sensitivity of more than $50 \%$ during the network rollout, which is a consequence of their direct impact on the number of required base stations. On the other hand, after ten years, the adoption and tariff setting, determining the revenues for the operator, are the most influencing parameters (together about 40\%). However, we still observe that the influence of the technical parameters remains significant at this time (together about $20 \%$ ), even in case of a five-year completion of the WiMAX network. This clearly stresses the importance of the technical parameters. Furthermore, adoption and tariffs will certainly influence each other, limiting their common influence on the NPV. 


\section{Sensitivity 5-year rollout, outdoor coverage}

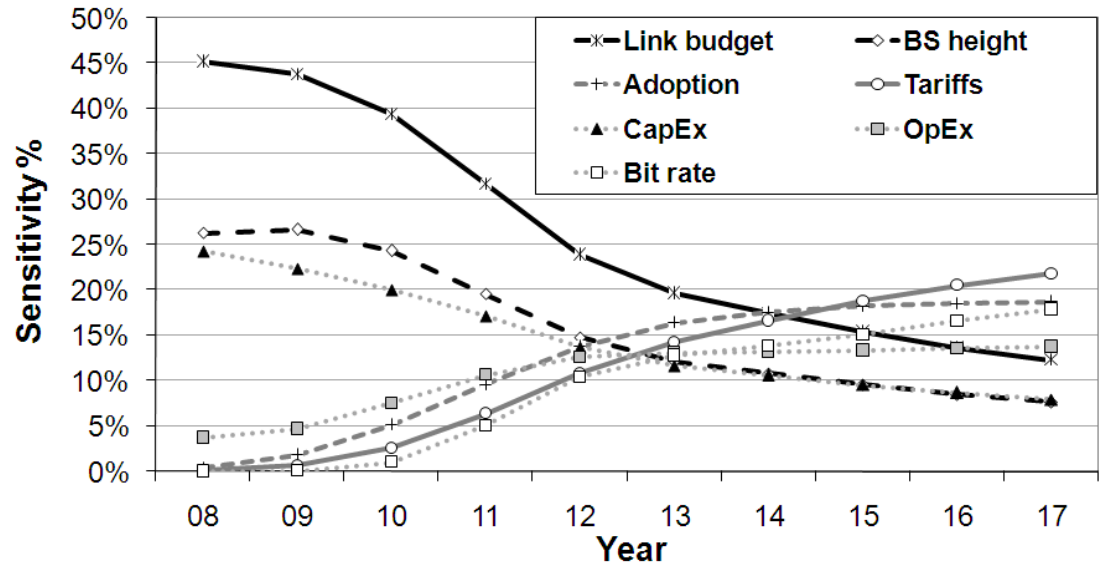

Fig. 5. NPV sensitivity results, taking into account technical as well as economic parameters.

\section{Conclusions}

In this paper, we have investigated the influence of technical improvements on the economic feasibility of a Mobile WiMAX deployment. Enhanced antenna technologies such as MIMO and beamforming lead to larger WiMAX ranges resulting in a reduction of the required number of base stations. In our business case for Belgium, we obtain a decrease of $30 \%$ of the number of base stations between our reference scenario and the most optimistic scenario taking into account MIMO as well as beamforming. Of course, this is very beneficial for the general feasibility of a Mobile WiMAX rollout. As we have assumed that the WiMAX network is gradually rolled out from dense urban areas to less populated ones, we can define an indication of the minimum required population density for making the business case feasible. For outdoor coverage in Belgium the most optimistic scenario reduces the required population density with $60 \%$, taking into account an adoption of ca. $15 \%$ of the households. The feasibility of the rollout of Mobile WiMAX also strongly depends on whether outdoor or indoor coverage is considered. To guarantee indoor coverage, a much larger number of base stations is required to cope with the indoor penetration loss. This leads to an increase by a factor of 4 to 5 of the required number of subscribers for making the rollout as feasible as the outdoor coverage business case (possible by a higher population density or a higher user adoption).

Both technical and economic parameters greatly influence the business case for a Mobile WiMAX rollout. The sensitivity analysis shows that the technical parameters have a significant influence exceeding the economic parameters during the rollout years (more than 50\%). After these years, economic parameters such as adoption and service tariffs are most determining, but the technical ones remain very important. When the feasibility of new technologies, such as Mobile WiMAX, is analyzed, one 
has to be very careful when selecting the values for technical parameters and especially the link budget parameters.

Acknowledgments. W. Joseph is a Post-Doctoral Fellow at the FWO-V (Research Foundation at Flanders).

\section{References}

1. IEEE Std. 802.16 - 2004, IEEE Standard for Local and Metropolitan area Networks: Part 16: Air Interface for fixed broadband wireless access systems. (2004)

2. ETSI: ETSI Broadband Radio Access Networks (BRAN) HIPERMAN Physical (PHY) layer. Standards TS 102 177. (2003)

3. IEEE Std. 802.16e - 2005, Amendment to IEEE Standard for Local and Metropolitan area Networks: Part 16: Air Interface for fixed broadband wireless access systems - Physical and Medium Access Control Layers for Combined Fixed and Mobile Operations in Licensed Bands. (2006)

4. WiMAX Forum, http://www.wimaxforum.org

5. WiMAX Forum White Paper: Mobile WiMAX-Part 1: A Technical Overview and Performance Evaluation. (2006)

6. Wang, F, Ghosh, A., Love, R., Stewart, K., Ratasuk, R., Bachul, R., Sun, Y., Zhao, Q.: IEEE 802.16e System Performance: Analysis and Simulations. In: 2005 IEEE 16th International Symposium on Personal, Indoor and Mobile Radio Communications (PIMRC2005), vol.2, pp. 900-904. Berlin (2005)

7. Hottinen, A., Kuusela, M., Hugl, K., Zhang, J., Raghothaman, B.: Industrial Embrace of Smart Antennas and MIMO. In: IEEE Wireless Communications Magazine, vol. 13, pp. 816 (2006)

8. Lannoo, B. et al: Business scenarios for a WiMAX deployment in Belgium. In: Proceedings of IEEE Mobile WiMAX 2007 conference, pp. 132-137. Orlando (2007)

9. Lannoo, B. et al: Economic Feasibility Study of a Mobile WiMAX Rollout in Belgium: Sensitivity Analysis and Real Options Thinking. In: Proceedings of Broadband Europe. Antwerp (2007)

10.WiMAX Forum White Paper: WiMAX System Evaluation Methodology, V1.0. (2007)

11.Nuaymi, L.: WiMAX: Technology for Broadband Wireless Access. Wiley (2007)

12.IEEE 802.16 Broadband Wireless Access Working Group: Simulation Results for Subchannelization. (2002)

13.Joseph, W., Martens, L.: Performance Evaluation of Broadband Fixed Wireless System based on IEEE 802.16. In: IEEE Wireless Communications and Networking Conference (WCNC2006), vol. 2, pp. 978-983. Las Vegas (2006)

14.Erceg, V. et al: An empirically based path loss model for wireless channels in suburban environments. In: IEEE J. Sel. Areas Commun., vol. 17, no. 7, pp. 1205-1211 (1999)

15.ETSI, TR 102377 v1.1.1: Digital Video Broadcasting (DVB); DVB-H Implementation Guidelines. (2005)

16.ECC report 33: The analysis of the coexistence of FWA cells in the $3.4-3.8 \mathrm{GHz}$ band. (2003) 